\title{
A GENERAL PHYSICAL APPROACH TO SOLITARY WAVE CONSTRUCTION FROM LINEAR SOLUTIONS
}

\author{
W. HEREMAN*, A. KORPEL and P.P. BANERJEE** \\ Department of Electrical and Computer Engineering, The University of Iowa, Iowa City, IA 52242, U.S.A.
}

Received 10 July 1984, Revised 26 November 1984

\begin{abstract}
We simplify the physical approach of constructing solitary wave solutions of nondissipative evolution and wave equations from the physical mixing of the real, rather than complex, exponential solutions of the linear equation, in two separate regions. In our new approach, we use mixing in one region only to construct a closed form for the solitary wave solution valid in both regions. Moreover, we extend the approach to deal with equations whose solutions (like tanh ${ }^{2}$-type) have a constant term in their expansion into real exponentials, and with equations whose linear part allows more than two exponential solutions. Finally, we also demonstrate the application of our technique to a typical dissipative equation, e.g., the Burgers equation.
\end{abstract}

\section{Introduction}

Solitary waves are constant-profile solutions of nonlinear dispersive evolution or wave equations. Physically, one may consider them to be a spectrum of harmonic waves traveling with the same velocity. These waves (each of which is composed of a pair of complex conjugate exponentials with imaginary exponents) are solutions of the underlying linear equation but would normally travel with different velocities due to dispersion. In some complicated manner the nonlinearity couples these waves by mixing and locks their velocities and phases so that they form a solitary wave. Such a model [1] has been called 'nonlinear coherent' [2].

Mathematical analyses using this model $[3,4]$ have been only partly successful owing to the extreme complexity arising from the mixing of the complex traveling wave components. In 1978, two, apparently little noticed, papers appeared

\footnotetext{
* On leave of absence from the Institute for Theoretical Mechanics, University of Gent, B-9000 Gent, Belgium.

** Present address: Department of Electrical and Computer Engineering, Syracuse University, Syracuse, NY 13210, U.S.A.
}

independently, dealing with the construction of solutions of nonlinear dispersive evolution and wave equations from traveling wave solutions of the linear equation. The first paper [5], by Rosales, is an extensive involved mathematical treatment which, starting from a purely formal iteration procedure, arrives a posteriori at the fact that the complete solution appears to be built up from the exponential traveling wave solutions of the linear equation and with imaginary exponents. The second paper [6], by Korpel, is physics oriented and starts a priori assuming, on physical grounds and a knowledge of the nature of the final solution, that such an expansion is not only possible, but the problem is made more tractable through the use of exponential solutions of the linear system with real, rather than imaginary, exponents.

In the linear system such solutions are, of course, not physical, unless they terminate on some boundary in the manner of, for instance, evanescent waves. In the nonlinear system, however, it turns out that this is no longer true: the left half of a pulse (consisting of rising exponentials) may, in fact, provide the boundary conditions for the right 
half (consisting of decaying exponentials) so that continuity is preserved at the center. The feasibility of the method lies in the fact that in the mathematical sense, it is far simpler to 'mix' real exponentials than harmonic functions built up from imaginary exponentials. In the original note [6], this was demonstrated for the $\mathrm{KdV}$ equation for which two such linear solutions, i.e., resp. $\exp ( \pm K \xi), K=$ $v^{1 / 2}, \xi=x-v t$, exist. The well-known $\operatorname{sech}^{2}$ solution was reconstructed by separate expansions for $\xi<0$ (rising exponentials: $\exp (n K \xi), n \in \mathbb{N}$ ) and $\xi>0$ (decaying exponentials: $\exp (-n K \xi)$ ), and finally imposing continuity conditions at $\xi=0$. The method has since been tested for a large number of evolution and wave equations, all having two linear solutions $(\exp ( \pm K \xi))$ and all leading to sech-type or $\operatorname{sech}^{2}$-type solitary wave solutions.

Subsequent investigations have revealed some new and intriguing facts which we report on in this paper by means of suitable examples. A more detailed and systematical approach to the general problem will be published later [7].

The present paper continues to emphasize the physics in order to demonstrate that it is also possible to use the harmonics of only one type of real exponential in order to arrive at the closed form of the $\mathrm{KdV}$ solution. The continuity conditions can then be dropped and the solution generalized by the inclusion of an arbitrary phase shift. This is, in fact, implicit in both the papers quoted before $[5,6]$ and will be clear from the result in Section 2.

Moreover, for this example, we consider other possible solutions, such as $\tanh ^{2} \hat{K} \xi$, which have a constant $(d c)$ term in their expansion with real exponentials. Now, solutions built up of the mixing of one kind of exponentials only (e.g., either decaying or rising) cannot have a constant term, $c_{1}$, in their expansion. How, then, do we construct such types of solutions? One approach would be to try and use all the linear solutions (decaying and rising exponentials), and consider their harmonic and mixing contributions, among which there would be a constant term. However, for the sake of simplicity, we choose to write the stationary solution $\phi$ of the nonlinear evolution (or wave) equation as $\phi=\hat{\phi}+c_{1}$, where $\hat{\phi}$ may, as before, be expanded in harmonics or mixing terms of one kind of exponentials (e.g., decaying or rising) having no constant term in its expansion into real exponentials. We therefore substitute $\hat{\phi}+c_{1}$ for $\phi$ in the nonlinear equation and consider the linear part of the resulting equation. In order for our technique to be successful, this linear part must now exhibit rising and decaying exponential solutions. This, in turn, imposes certain conditions on $c_{1}$ so that we can, after building up $\hat{\phi}$, find $\phi$ itself. In Section 2 , where we demonstrate this for the KdV equation, it turns out that we find a general solution of the form $c_{1}+c_{2} \operatorname{sech}^{2} \hat{K} \xi$, from which the well-known $\operatorname{sech}^{2}$ solution and the 'well'-type $\tanh ^{2}$ solution follow as special cases, through proper choice of the constants $c_{1}$ and $c_{2}$.

Secondly, in Section 3, we treat the case of the $\mathrm{KdV}$ equation with an additional fifth-order dispersive term $[8,9]$, where there exist four real solutions, $\exp \left( \pm K_{1,2} \xi\right)$, of the linear equation. By using only the two decaying exponentials, e.g., $\exp \left(-K_{1,2} \xi\right)$, and imposing conditions of commensurability of the mixing terms, we again find a series that accurately represents the $\operatorname{sech}^{4}$ solution [8].

Thirdly, we treat the case of a dissipative equation, e.g., the Burgers equation [10], where only one solution, $\exp (-K \xi), K=v$, exists for the linear part of the equation. As before, we find that the correct closed form kink-type solution can be built up from the harmonics of the one decaying exponential solution. However, as we will show, the physical interpretation in terms of mixing and harmonics is considerably different from that given for the case of the nondissipative equations treated before.

Although we do not claim exclusive novelty for all of the formal method as such, we do believe that our independently developed physical approach to the problem will contribute greatly to the physical insight developed by using such concepts as 'harmonics', 'finite real exponentials', 'mixing' etc, familiar to engineers and physicists. 
In this regard, we feel that our technique using real exponential solutions of the underlying linear equation provides a complementary and, perhaps, more physical and less mathematically formidable, approach to the otherwise complicated problem. For an exhaustive mathematical treatment starting from complex exponentials, and a comparison between the two points of view, the reader is referred to Rosales' paper [5].

\section{General solution of the $\mathrm{KdV}$ equation}

In this section we demonstrate how to construct solutions of nonlinear equations that contain a constant term in their expansion, using the $\mathrm{KdV}$ equation $[11,12]$

$$
u_{t}+\alpha u u_{x}+u_{3 x}=0, \quad \alpha \in \mathbb{R},
$$

as an example.

In our search for stationary solutions for (1), we introduce a traveling frame of reference $\xi=x-v t$ to transform the PDE in (1) into an ODE in $\phi(\xi) \triangleq u(x, t)$ where $v$ is the anticipated traveling wave velocity, and integrate (largely for mathematical convenience) with respect to $\xi$ to get

$$
-v \phi+(\alpha / 2) \phi^{2}+\phi_{2 \xi}+c_{1} C=0
$$

where $c_{1} C$ is an integration constant. As we shall see below, the purpose of this integration constant is to facilitate exponential solutions for the linear part of the transformed equation in $\hat{\phi}$ :

$$
\phi=c_{1}+\hat{\phi}
$$

where $c_{1}$ is a constant. Indeed, substitution of (3) in (2) yields

$$
\begin{aligned}
& \left(\alpha c_{1}-v\right) \hat{\phi}+(\alpha / 2) \hat{\phi}^{2}+\hat{\phi}_{2 \xi} \\
& \quad+\left(-v c_{1}+(\alpha / 2) c_{1}^{2}+c_{1} C\right)=0
\end{aligned}
$$

the linear part of which has two exponential solutions of the form $\exp ( \pm K \xi)$ with

$$
K^{2}=v-\alpha c_{1}=2 C-v,
$$

iff the $\hat{\phi}$-independent part in (4) is set equal to zero; i.e.,

$$
c_{1}=2(v-C) / \alpha .
$$

Using (5) and (6), the transformed equation (4) becomes

$$
(v-2 C) \hat{\phi}+(\alpha / 2) \hat{\phi}^{2}+\hat{\phi}_{2 \xi}=0 .
$$

We now seek stationary solutions of $\hat{\phi}$ in terms of the harmonics of, say, decaying exponentials. To this end, we scale $\hat{\phi}$ according to

$$
\hat{\phi}=(2 / \alpha)(2 C-v) \tilde{\phi},
$$

and expand $\tilde{\phi}$ in terms of the harmonics of the decaying exponential solution to the linear equation:

$$
\begin{aligned}
& \tilde{\phi}=\sum_{n=1}^{\infty} a_{n} g^{n}(\xi), \\
& g(\xi)=\exp (-K \xi) .
\end{aligned}
$$

We next substitute (8)-(10) into (7) to get the recursion relation

$$
\begin{aligned}
& \left(n^{2}-1\right) a_{n}+\sum_{l=1}^{n-1} a_{l} a_{n-1}=0, \\
& n \geqslant 2, a_{1} \text { arbitrary, }
\end{aligned}
$$

where use has been made of (5) and (6) to simplify. The $a_{n}$ 's are then given by [6]:

$$
a_{n}=6 n(-1)^{n+1}\left(a_{1} / 6\right)^{n}, \quad a_{1}>0,
$$

so that with (3), (6), (8) and (9), the closed form solution $\phi$ may be written as:

$$
\begin{aligned}
\phi= & (2 / \alpha)(v-C) \\
& +(2 / \alpha)(2 C-v) 6 a g /(1+a g)^{2}, \\
a= & a_{1} / 6 .
\end{aligned}
$$

Note that the closed form (13) for $\phi$ has been built up from a convergent power series of decaying exponentials $g(\xi)$ for $a g<1$ (i.e., in the region $\left.\xi>\xi_{0}, \quad \xi_{0}=(\ln a) / K\right)$. However, (13) is also expressible as a convergent power series in $(a g)^{-1}$ for $a g>1$ (i.e., in the region $\xi<\xi_{0}$ ) where $g^{-1}$ ( $=\exp (K \xi))$ is a (bounded) rising exponential 
solution to the linear equation. Since (13) is continuous at $a g=1$, it is, therefore, a valid solution over the entire region $-\infty<\xi<\infty$. Physically speaking, this means that the solution in the region $\xi \geqslant \xi_{0}$ (built up from harmonics of decaying exponentials) provides the boundary conditions for the solution in the region $\xi \leqslant \xi_{0}$ (built up from harmonics of rising exponentials) ensuring continuity at $\xi=\xi_{0}$. The final solution $u(x, t)$ may now be expressed, using (5), (10) and (13), as

$$
\begin{aligned}
u(x, t)= & (2 / \alpha)(v-C)+(3 / \alpha) \\
& \times(2 C-v) \operatorname{sech}^{2}\left\{\frac{1}{2}(2 C-v)^{1 / 2}\right. \\
& \times(x-v t)+\delta\},
\end{aligned}
$$

where $\delta\left(=\frac{1}{2} \ln (1 / a)\right)$ represents an arbitrary phase factor.

As special cases, note that the choice of the constant $C$ as $v$ and $v / 4$, respectively lead to the well-known $\operatorname{sech}^{2}$ solution $[11,12]$

$$
\begin{aligned}
& u(x, t) \\
& \quad=(3 v / \alpha) \operatorname{sech}^{2}\left\{\frac{1}{2} v^{1 / 2}(x-v t)+\delta\right\},
\end{aligned}
$$

and a 'well'-type $\tanh ^{2}$ solution

$$
\begin{aligned}
& u(x, t) \\
& \quad=(3 v / 2 \alpha) \tanh ^{2}\left\{\frac{1}{2}(-v / 2)^{1 / 2}(x-v t)+\delta\right\} .
\end{aligned}
$$

In passing, it is interesting to observe that the solution $u(x, t)$ given in (14) contains a constant term and a sech ${ }^{2}$-type term, both of which are individually solutions of the $\mathrm{KdV}$ equation. The general solution $u$, given by (14), may therefore be visualized as the superposition of two particular solutions of the $\mathrm{KdV}$ equation of appropriate amplitudes which are now locked together with a different velocity $\left(2 C-v_{\text {as }}\right)$, where $v_{\text {as }}$ is the velocity of the free (associated) $\mathrm{KdV}$ soliton. This is similar to the model of cnoidal wave decomposition outlined in [13] where it has been shown that the cnoidal wave $\left(c n^{2}\right)$ solution of the $\mathrm{KdV}$ equation may be thought of as consisting of an infinite number of $\mathrm{KdV}$ solitons that have become locked in (reduced) velocity. The analogy of the decompositions in these limiting cases to soliton interaction in general, though intriguing to speculate on, is outside the scope of this paper.

\section{The KdV equation with additional fifth-order dispersive term}

As a second illustration of the applicability of our modified technique, we now solve a nonlinear evolution equation, e.g., the $\mathrm{KdV}$ equation with an additional fifth-order dispersive term $[8,9]$

$$
u_{t}+\alpha u u_{x}+\beta u_{3 x}+u_{5 x}=0, \quad \alpha, \beta \in \mathbb{R},
$$

which has four real solutions, $\exp \left( \pm K_{1,2} \xi\right)$, for its linear part. Since our objective in this section is to primarily demonstrate the applicability of our technique to equations whose linear part allows more than two real exponential solutions, we will, for the sake of simplicity, only look for stationary solutions that have no constant term in their expansion into real exponentials. The technique may be readily applied to find more general solutions by proceeding along the guidelines outlined in Section 2.

In order to find stationary traveling wave solutions to (17), we define $\phi(\xi) \triangleq u(x, t), \xi=$ $x-v t$, substitute in (17) and integrate with respect to $\xi$ to obtain

$$
-v \phi+(\alpha / 2) \phi^{2}+\beta \phi_{2 \xi}+\phi_{4 \xi}=0,
$$

where we have now neglected the integration constant, since in this example, we are not interested in solutions having a constant term in their expansion into real exponentials. Real solutions of the linear part of (18) may be represented as $\exp \left( \pm K_{1,2} \xi\right)$ in which

$$
\begin{aligned}
& K_{1,2}=\left[\left\{-\beta \pm\left(\beta^{2}+4 v\right)^{1 / 2}\right\} / 2\right]^{1 / 2}, \\
& \beta<0,-\beta^{2} / 4<v<0 .
\end{aligned}
$$

Physically speaking, therefore, we would expect the final solution $\phi(\xi)$ to be representable in terms of the harmonics and mixing terms of the two 


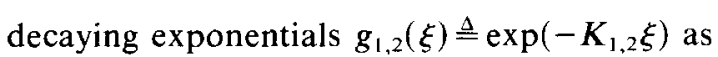

$$
\phi(\xi) \propto \sum_{\substack{n_{1}=0 \\\left(n_{1}, n_{2}\right) \neq\{0,0\}}}^{\infty} \sum_{\substack{n_{2}=0 \\ n_{1} n_{2}}}^{\infty} g_{1}^{n_{1}}(\xi) g_{2}^{n_{2}}(\xi)
$$

Alternatively, and for the sake of mathematical simplicity, we now write $\phi \propto \sum_{n=1}^{\infty} a_{n} g^{n}$ with

$$
g(\xi) \triangleq \exp (-K \xi)
$$

and seek for integral values $M_{1,2}$ satisfying

$$
K=K_{1} / M_{1}=K_{2} / M_{2},
$$

anticipating that the final solution may be built up as the sum of the powers of only one decaying exponential, as in (9), commensurate with all possible mixing terms and powers of the two decaying exponentials. In fact, as a check, we will later reexpress our final result in a way analogous to (20) to explicitly demonstrate the mixing terms between the two linear solutions $g_{1}$ and $g_{2}$.

For computational convenience that will become clear later, we introduce

$$
\phi=-(v / 18 \alpha) \tilde{\phi}
$$

and substitute (9) and (23) into (18) to get

$$
\begin{aligned}
& \sum_{n=1}^{\infty}\left(n^{4} K^{4}+n^{2} \beta K^{2}-v\right) a_{n} g^{n} \\
& -(v / 36) \sum_{n=2}^{\infty} \sum_{l=1}^{n-1} a_{l} a_{n-l} g^{n}=0 .
\end{aligned}
$$

It can be shown [7] that the choice

$$
\begin{aligned}
& v=-36 \beta^{2} / 169, \\
& K=(-\beta / 13)^{1 / 2}
\end{aligned}
$$

ensures the commensurability expressed by (22) with

$$
\begin{aligned}
& K_{1}=2(-\beta / 13)^{1 / 2}, \\
& K_{2}=3(-\beta / 13)^{1 / 2},
\end{aligned}
$$

so that the second and third harmonics of $g$, which are legitimate solutions of the linear part of (18), build up the final solitary wave solution through harmonic generation and mixing. The recursion relation following from (24) may, in this case, be expressed canonically as

$$
\begin{aligned}
& \left(n^{2}-4\right)\left(n^{2}-9\right) a_{n}+\sum_{l=1}^{n-1} a_{l} a_{n-l}=0, \\
& n \geqslant 2, a_{1}=0,
\end{aligned}
$$

from which the general structure of $a_{n}$ may be calculated as [7]

$$
\begin{aligned}
& a_{n}=140(-1)^{n+1} n\left(n^{2}-1\right) a^{n}, \\
& a=-a_{3} / 4 a_{2}>0 .
\end{aligned}
$$

Using (29) in (9), $\tilde{\phi}$ may be expressed in closed form as

$$
\tilde{\phi}=-840(a g)^{2} /(1+a g)^{4} .
$$

By reasoning as in Section 2, it may be readily verified that $(30)$ represents a valid solution over the entire region $-\infty<\xi<\infty$. The final solution for $\phi$, and hence for $u$, after denormalization using (23), therefore reads

$$
\begin{aligned}
u(x, t) \triangleq & \phi(x-v t) \\
= & -\left(105 \beta^{2} / 169 \alpha\right) \\
& \times \operatorname{sech}^{4}\left\{\frac{1}{2}(-\beta / 13)^{1 / 2}\right. \\
& \left.\times\left(x+\left(36 \beta^{2} / 169\right) t\right)+\delta\right\}, \\
\delta=\frac{1}{2} \ln 1 / a &
\end{aligned}
$$

In retrospect, note that the series (9), with (29), may be reexpressed as

$$
\tilde{\phi}(\xi)=\sum_{n_{1}=0}^{\infty} \sum_{n_{2}=0}^{\infty} a_{n_{1} n_{2}} g_{1}^{n_{1}}(\xi) g_{2}^{n_{2}}(\xi),
$$

with

$$
g_{1}=g^{2}, \quad g_{2}=g^{3},
$$

and

$$
\begin{aligned}
& a_{10}=a_{2}, \quad a_{01}=a_{3}, \quad a_{20}=-a_{2}^{2} / 84, \\
& a_{02}=a_{2}^{3} / 36288, \quad a_{11}=-a_{2} a_{3} / 168, \text { etc. }
\end{aligned}
$$

which is analogous to $(20)$, demonstrating mixing between $g_{1}$ and $g_{2}$, as claimed earlier. 


\section{The Burgers equation}

Thus far, we have used our technique to solve nonlinear nondissipative evolution equations whose solutions have a constant term in their expansion into real exponentials or whose linear part allows more than two real exponential solutions. The technique may also be readily used to construct solutions of nonlinear nondissipative wave equations in a similar way [7]. To test the applicability of the method to nonlinear dissipative equations, we shall now attempt to solve the Burgers equation, which is the simplest evolution equation combining nonlinearity and diffusion and is expressible in the form [10]:

$$
u_{t}+\alpha u u_{x}-u_{2 x}=0, \quad \alpha \in \mathbb{R},
$$

Analogous to the way (18) was derived from (17), we may transform (35) into

$$
-v \phi+(\alpha / 2) \phi^{2}-\phi_{\xi}=0
$$

where, as in Section 3, we are only interested in solutions having no constant term in their expansion into real exponentials.

As noted in the Introduction, only one real exponential solution $g(\xi) \triangleq \exp (-K \xi),-\infty<\xi<$ $\infty$, with

$$
K=v
$$

exists for the linear part of (36).

For mathematical convenience once again, we introduce the scale

$$
\phi=(2 v / \alpha) \tilde{\phi},
$$

and substitute (9) and (38) in (36) to derive the recursion relation

$$
\begin{aligned}
& (n-1) a_{n}+\sum_{l=1}^{n-1} a_{l} a_{n-l}=0, \\
& n \geqslant 2, a_{1} \text { arbitrary, }
\end{aligned}
$$

from which the general structure of $a_{n}$ may be readily recognized as

$$
a_{n}=(-1)^{n+1} a_{1}^{n}, \quad a_{1}>0 .
$$

Using (40) in (9), $\tilde{\phi}$ may be expressed in closed form as [7]:

$$
\begin{aligned}
\tilde{\phi} & =a_{1} g /\left(1+a_{1} g\right) \\
& ={ }_{2}^{1}\left[1-\left(1-a_{1} g\right) /\left(1+a_{1} g\right)\right] .
\end{aligned}
$$

As before, note that the closed form (41) for $\tilde{\phi}$ has been built up from a convergent power series of decaying exponentials $g(\xi)$ for $a g<1$ (i.e., in the region $\left.\xi>\xi_{0}, \xi_{0}=(\ln a) / K\right)$. However, unlike the cases treated in Sections 2 and 3 , the expansion of (41) in powers of $(\mathrm{ag})^{-1}$ is physically irrelevant, since $g^{-1}(=\exp (K \xi))$ is no longer a solution of the linear equation. The physical picture of matching solutions built up from the harmonics of decaying $(\exp (-K \xi))$ and rising $(\exp (K \xi))$ exponentials for the case of nondissipative evolution and wave equations is no longer achievable. The solution in the region $\xi<\xi_{0}(a g>1)$ can thus be built up only from decaying exponentials, and the series solution in powers of $g$ is no longer convergent. We feel the reason for this is the fact that the steady-state attainment can only be achieved through a balance between a continuous supply of energy and the dissipation in the system, which is finally responsible for the boundedness of the closed form solution (41). Use of (37) and subsequent denormalization of (41) using (38) finally yields the steady-state solution (attainable as $t \rightarrow \infty[10]$ ):

$$
\begin{aligned}
& u(x, t)=(v / \alpha)[1-\tanh \{(v / 2)(x-v t)+\delta\}], \\
& \delta=\frac{1}{2} \ln 1 / a,
\end{aligned}
$$

which is the familiar kink-type Taylor shockprofile solution [11].

\section{Conclusion}

In summary, we have presented a simplified physical picture for the construction of solitary waves using the underlying concepts of harmonic generation and mixing of the real exponential solutions of the linear equation. The emphasis on the physics in our method complements the more formal mathematical treatment presented else- 
where [5]. A more elaborate account of our method containing guidelines towards solving the recursion relations that arise will be presented later [7].

\section{Acknowledgment}

This research was supported by NATO under a Research Fellowship Program awarded to W.H. and by the National Science Foundation under Grant No. ECS81-21781.

\section{References}

[1] A. Korpel, "Solitary wave formation through $m$-th order parametric interaction", Proc. IEEE 67, 1442-1443 (1979).

[2] H.C. Yuen and B.M. Lake, "Nonlinear wave concepts applied to deep-water waves", in: K. Lonngren and A. Scott, eds., Solitons in Action, Academic Press, New York (1978).

[3] A. Korpel, "A frequency approach to nonlinear dispersive waves", J. Acoust. Soc. Amer. 70, 1954-1958 (1980).
[4] P.P. Banerjee and A. Korpel, "Stability of acoustic nonlinear dispersive eigenmodes", J. Acoust. Soc. Amer. 70, 157-164 (1981).

[5] R. Rosales, "Exact solutions of some nonlinear evolution equations", Stud. Appl. Math. 59, 117-151 (1978).

[6] A. Korpel, "Solitary wave formation through nonlinear coupling of finite exponential waves", Phys. Lett. 68A, 179-181 (1978).

[7] W. Hereman, P.P. Banerjee, A. Korpel, G. Assanto and A. Van Immerzeele, "Exact solitary wave solutions of nonlinear evolution and wave equations using a direct algebraic method", in preparation.

[8] Y. Yamamoto and E. Takizawa, "On a solution of a nonlinear time-evolution equation of fifth order", $J$. Phys. Soc. Japan 50, 1421-1422 (1981).

[9] Y. Kodama and T. Tanuiti, "Higher-order approximation in the reductive perturbation method I and II", $J$. Phys. Soc. Japan 45, 298-314 (1978).

[10] G.B. Whitham, Linear and Nonlinear Waves, Wiley, New York (1974).

[11] R.K. Dodd, J.C. Eilbeck, J. Gibbon and H. Morris, Solitons and Nonlinear Wave Equations, Academic Press, New York (1982).

[12] A.C. Scott, F.Y.F. Chu and D.W. McLaughlin, "The soliton: a new concept in applied science", Proc. IEEE 61, 1443-1483 (1973).

[13] A. Korpel and P.P. Banerjee, "Exact decomposition of cnoidal waves into associated solitons", Phys. Lett. $82 \mathrm{~A}$, 113-115 (1981). 